\title{
Editorial
}

\section{Clinical Research to Standard of Care: When Does the Transition Occur?}

\author{
Paul H. Sugarbaker, MD, FACS, FRCS
}

The steps for clinical research to mature into a standard of care have never been well defined. Adding to the dilemma are different criteria among oncologic disciplines. Medical oncologists may insist on multi-institutional phase III trials before adopting a new treatment. Oncologic surgeons may not require this type A statistical data. For example, a new treatment, surgical resection of liver metastases from colorectal cancer, is currently standard of care in selected patients. No randomized data exist for resection versus no resection.

A general sequence commonly used for this transition involves an evaluation of efficacy, morbidity, mortality, and cost. Comparisons of the new treatment with other plans that are currently accepted as standard of care are helpful in making these decisions.

Carcinomatosis is an area of clinical research that may need to move to standard of care. Of course, as with any other surgical treatment, knowledgeable selection of patients who will profit most is a necessity. Efficacy regarding carcinomatosis treatment with curative intent has been repeatedly demonstrated. The first and most widely published set of data concerns minimally aggressive mucinous appendiceal tumors with peritoneal dissemination that are treated with cytoreductive surgery combined with perioperative intraperitoneal chemotherapy. In 1999, Sugarbaker ${ }^{1}$ and Chang showed an $80 \%$ 5-year actuarial survival in 250 patients who had a complete cytoreduction of appendiceal malignancy. ${ }^{1}$ Mansfield $^{2}$ reported his own 5-year actuarial survival of more than $75 \%$. Witkamp et al. ${ }^{3}$ reported a 3 -year actuarial survival of $81 \%$. Gough et al., ${ }^{4}$ from the Mayo Clinic, emphasize

Received June 3, 2003; accepted August 22, 2003.

From the Washington Cancer Institute, Washington, DC.

Address correspondence to: Paul H. Sugarbaker, MD, FACS, FRCS, Washington Cancer Institute, 110 Irving St., NW, Washington, DC 20010; Fax: 202-877-8602; E-mail: Paul.Sugarbaker@medstar.net.

Published by Lippincott Williams \& Wilkins (C) 2003 The Society of Surgical Oncology, Inc. that long-term follow-up is necessary in this group of patients.

Similar benefit with comparison to historical controls has been reported with another rare disease, malignant peritoneal mesothelioma. In contrast to a median survival of approximately 9 months reported in the literature, aggressive cytoreduction with intraperitoneal chemotherapy resulted in a median survival of 67 months in 68 people treated at the Washington Cancer Institute and in 92 months in 49 patients treated at the National Institutes of Health. 5

Concerning a much more common disease, colorectal cancer, Glehen and Sugarbaker ${ }^{6}$ collected data on 333 patients from five different institutions treated by cytoreductive surgery and intraperitoneal chemotherapy. The 3-year survival was $31 \%$ (range, $23 \%$ to $47 \%$ ).

Undoubtedly, the most important paper concerning colorectal carcinomatosis is the randomized trial from Amsterdam reported by Zoetmulder et al. ${ }^{7}$ A 16\% 2-year survival was seen in patients treated by systemic chemotherapy. A highly statistically significant improvement to $43 \%$ was seen in the group treated by the combined modality of cytoreduction plus intraperitoneal chemotherapy $(P=.0145)$.

The report by Glehen et al. ${ }^{8}$ from Lyon helps to establish the second important requirement for the transition of clinical research to standard of practice. In 216 consecutive procedures, they show a mortality rate of $3.2 \%$ and a morbidity rate of $24.5 \%$. These data conform to that which is expected for other major surgical procedures in gastrointestinal cancer.

As might be expected, morbidity of the combined procedure (i.e., cytoreductive surgery and hyperthermic intraperitoneal chemotherapy) increased with the extent of the surgery. Duration of surgery, number of anastomoses required, and number of peritonectomy procedures had a significant impact on the incidence of complications. 
The safety of the intraperitoneal chemohyperthermia at Lyon was remarkable. In their analysis of the causes of mortality in seven patients, only one death was clearly related to the use of hyperthermic intraperitoneal chemotherapy. The hematologic toxicity they noted was only $4.6 \%$. The hematologic toxicities can usually be prevented through patient selection. Glehen et al. ${ }^{8}$ point out that stem cell damage from extensive prior systemic chemotherapy was probable in 8 of the 10 patients who developed hematologic toxicity. Schnake et al. ${ }^{9}$ suggest the same. The generous use of dose reductions for patients who may be especially vulnerable to chemotherapy toxicity is necessary. Remember, this chemotherapy is not only regional, it is absorbed almost in entirety and can cause profound bone marrow suppression.

One can rightly ask the question: "What should be done with my patient in mid-year 2003 who has low volume peritoneal seeding from a gastrointestinal malignancy?" Has the transition from clinical research to standard of practice occurred in this group of patients? In my opinion, the answer is "yes," but only under very strict conditions: There must be low volume seeding (pseudomyxoma and peritoneal mesothelioma excluded). The cytoreduction must be complete or near complete. The team performing this treatment strategy must be sufficiently experienced. Numerous peritoneal surface malignancy treatment centers operating over the last two decades have established the importance of proper patient selection, meticulous peritonectomy technique, standardized perioperative chemotherapy regimens, and knowledgeable, experienced postoperative care. The responsibilities of a surgeon as an oncologist can be redefined as surgeons who must employ intraperitoneal and intrapleural chemotherapy as part of standard surgical practice. Surgeons reading this editorial and wishing to become involved in this new gastrointestinal cancer treatment must be aware that a learning curve exists, not only for cytoreductive surgery, but also for the safe administration of maximal amounts of hyperthermic intraperitoneal chemotherapy. For efficacy to be recognized, morbidity and mortality must be maintained at a minimum.

Glehen et al. ${ }^{8}$ have given us all a standard of excellence to pursue in the movement of this clinical research project into its new position as a standard of care.

\section{REFERENCES}

1. Sugarbaker PH, Chang D. Results of treatment of 385 patients with peritoneal surface spread of appendiceal malignancy. Ann Surg Oncol 1999;6:727-31.

2. Mansfield PF. Appendiceal malignancy: Where do we stand? Ann Surg Oncol 1999;6:715-6.

3. Witkamp AJ, de Bree E, Kaag MM, et al. Extensive surgical cytoreduction and intraoperative hyperthermic intraperitoneal chemotherapy in patients with pseudomyxoma peritonei. $\mathrm{Br} \mathrm{J} \mathrm{Surg}$ 2001;88:458-63.

4. Gough DB, Donohue JH, Schutt AJ, et al. Pseudomyxoma peritonei. Long-term patient survival with an aggressive regional approach. Ann Surg 1994;219:112-9.

5. Carroll NM, Mohamed F, Sugarbaker PH, Alexander HR. Surgery, hyperthermic chemoperfusion, and postoperative chemotherapy: the National Cancer Institute and Washington Hospital Center experience. In: Pass HI, Vogelzang NJ, Carbone M, eds. Malignant mesothelioma: advances in pathogenesis, diagnosis and translational therapies. New York: Springer-Verlag Publishers (in press).

6. Glehen O, Sugarbaker PH. New perspectives in the management of colorectal cancer; what about peritoneal carcinomatosis? Scand J Surg 2003;92:178-9.

7. Zoetmulder FAN, Verwaal V, Ruth S. Hyperthermic intra peritoneal chemotherapy (HIPEC) with mitomycin C significantly improves survival in patients with peritoneal carcinomatosis of colorectal origin. J Clin Oncol 2003 (in press).

8. Glehen O, Osinsky D, Cotte E, et al. Intraperitoneal chemohyperthermia using a closed abdominal procedure and cytoreductive surgery for the treatment of peritoneal carcinomatosis: morbidity and mortality analysis of 216 consecutive procedures. Ann Surg Oncol 2003;10:863-9.

9. Schnake KJ, Sugarbaker PH, Yoo D. Neutropenia following perioperative intraperitoneal chemotherapy. Tumori 1999;85:41-6. 